Revista Brasil. Bot., V.32, n.1, p.183-188, jan.-mar. 2009

Scientific Note/Nota Científica

\title{
Efeitos alelopáticos de extratos aquosos de Crinum americanum $\mathrm{L}$.
}

\author{
JOSÉ PEDRO N. RIBEIRO ${ }^{1}$, REGINALDO S. MATSUMOTO ${ }^{1}$, LEANDRO K. TAKAO${ }^{1}$, \\ VALQUÍRIA M. VOLTARELLI ${ }^{1}$ e MARIA INÊS S. LIMA ${ }^{2,3}$
}

(recebido: 03 de abril de 2008; aceito: 11 de dezembro de 2008)

\begin{abstract}
Allelopathic effects of aqueous extracts of Crinum americanum L.). In this paper we investigated the action of the allelopathic properties of aqueous extracts of Crinum americanum L. on the percentage and average time of germination and initial growth of Lactuca sativa L., Sesamum indicum L. and Raphanus sativus L. and of the weed species: Echinochloa crusgalli (L.) P. Beauv., Ipomoea grandifolia (Dammer) O’Donell and Bidens pilosa L. We prepared aqueous extracts from individuals of $C$. americanum from a blind estuary in Caraguatatuba, São Paulo and set the osmotic control with PEG 6000. We analyzed the germination data with Kruskal-Wallis and Dunn's post test, and the initial growth data with ANOVA and Tukey's post test. For all the tested species the result we found out was a decrease in the germination and in the initial growth and an improvement of germination average time. The osmotic control indicated little influence of osmolarity on the tests. We concluded that $C$. americanum has allelopathic potential over the tested species.
\end{abstract}

Key words - allelopathy, macrophytes, Massaguaçu River, weed control

RESUMO - (Efeitos alelopáticos de extratos aquosos de Crinum americanum L.). Investigamos a ação de extratos aquosos de Crinum americanum L. sobre a germinação e crescimento inicial de Lactuca sativa L., Sesamum indicum L. e Raphanus sativus L. e das espécies invasoras: Echinochloa crusgalli (L.) P. Beauv., Ipomoea grandifolia (Dammer) O’Donell e Bidens pilosa L. Preparamos os extratos aquosos a partir de exemplares de um estuário cego em Caraguatatuba, São Paulo. Montamos controles osmóticos com PEG 6000. Analisamos os dados de germinação com Kruskal-Wallis e pós-teste de Dunn, e os dados de crescimento inicial com ANOVA e pós-teste de Tukey. Para todas as espécies testadas obtivemos diminuição na porcentagem e no crescimento inicial e aumento do tempo médio da germinação. O controle osmótico indicou pouca influência da osmolaridade na germinação. Concluímos que $C$. americanum possui potencial alelopático sobre as espécies testadas.

Palavras-chaves - alelopatia, macrófitas, plantas daninhas, Rio Massaguaçu

\section{Introdução}

Diversas plantas aquáticas produzem aleloquímicos. A liberação destes pelas macrófitas parece ser uma resposta evolutiva eficiente, contra o fitoplâncton e epífitas (Gross 2003, Erhard \& Gross 2006), uma vez que o sombreamento causado por estes é o principal fator limitante das macrófitas submersas (Phillips et al. 1978). Hilt (2006), em uma revisão dos efeitos alelopáticos de macrófitas sobre cianobactérias e algas, propôs que, em muitos casos, ocorre algum tipo de inibição. Huang et al. (1999) observaram que extratos de aguapé (Eichornia crassipes Solms) têm propriedades alelopáticas.

Crinum americanum L. (Amaryllidaceae) é uma macrófita que apresenta grande dominância em diversos

\footnotetext{
1. Programa de Pós-Graduação em Ecologia e Recursos Naturais, Universidade Federal de São Carlos, SP, Brasil.

2. Universidade Federal de São Carlos, Departamento de Botânica, Rodovia Washington Luiz, km 235, Caixa Postal 676, 13565-905 São Carlos, SP, Brasil.

3. Autor para correspondência: ines@ power.ufscar.br
}

locais (Mayer et al. 1998, Oliveira \& Ribeiro-Costa 2006), sugerindo que esta espécie possui atividades alelopáticas.

Diversos autores (Putnam 1983, Santos \& Rezende 2008) demonstraram a possibilidade de controlar espécies invasoras através de aleloquímicos. Os compostos naturais têm várias vantagens sobre os compostos sintéticos, como maior solubilidade em água, ausência de moléculas halogenadas e menor meia vida (Duke et al. 2000). É cada vez maior a necessidade de descoberta de novos herbicidas, visto que o número de espécies de ervas invasoras resistentes aos compostos tradicionais cresce a cada dia (Inderjit \& Bhowmik 2002).

$\mathrm{O}$ crescimento rápido e a rusticidade de $C$. americanum facilitam seu plantio, manutenção e reprodução em tanques colocando-o como uma boa possibilidade de planta doadora de herbicidas naturais em escala. Não localizamos nenhum trabalho sobre alelopatia para essa espécie. No entanto, estudos mostram a existência de flavanas e alcalóides (Ali et al. 1986, Ali et al. 1988, Trimino et al. 1987). Garuccio \& Arrigoni (1989) 
encontraram licorina em $C$. americanum, um alcalóide tido como forte inibidor da germinação.

Investigamos o potencial alelopático de extratos aquosos de $C$. americanum em diferentes concentrações sobre a porcentagem e tempo médio de germinação e sobre o crescimento inicial de espécies olerícolas bioindicadoras e de espécies invasoras de culturas.

\section{Material e métodos}

Realizamos este trabalho em duas fases distintas. $\mathrm{Na}$ primeira (fase I) submetemos as espécies olerícolas (Lactuca sativa L. (alface), Sesamum indicum L. (gergelim) e Raphanus sativus L. (rabanete)), utilizadas como indicadoras, aos extratos de $C$. americanum. Uma vez detectadas propriedades alelopáticas, iniciamos a segunda fase (fase II), constituída pelos ensaios dos extratos em espécies invasoras de culturas (Bidens pilosa L. (picão-preto), Echinochloa crusgalli (L.) P. Beauv. (capim-arroz) e Ipomoea grandifolia (Dammer) O’Donell (corda-de-viola)).

De forma assistemática coletamos indivíduos de $C$. americanum no estuário do Rio Massaguaçu, Caraguatatuba, SP. Dividimos 10 indivíduos em raiz, folhas e bainha das folhas. Trituramos o material na proporção $3 / 4(\mathrm{p} / \mathrm{p})$ que estabelecemos como extrato $100 \%$. Filtramos a vácuo, papel filtro $\left(80 \mathrm{~g} \mathrm{~m}^{-2}, 205 \mu \mathrm{m}\right)$ e fizemos outras diluições em água $(75 \%, 50 \%, 25 \%$ e $12,5 \%)$. Determinarmos a relação massa fresca/seca de cada parte $\left(\right.$ à $60{ }^{\circ} \mathrm{C}, 48 \mathrm{~h}$ ).

A fase I mostrou que a folha é o órgão mais ativo e a utilização de massa seca é a que possibilita uma melhor comparação com outros trabalhos. Assim, na fase II utilizamos apenas as folhas, secas à $40^{\circ} \mathrm{C}$, por 48 horas. Trituramos o material, homogeneizamos o pó em água destilada nas proporções $1 \%$ e $5 \%$, e depois filtramos o extrato a vácuo em papel filtro $\left(80 \mathrm{~g} \mathrm{~m}^{-2}, 205 \mu \mathrm{m}\right)$.

Ensaio de germinação - Esterilizamos placas de Petri $(9 \mathrm{~cm})$ forradas com papel filtro. Distribuímos 30 sementes na placa pré umedecida com $5 \mathrm{~mL}$ do tratamento. Colocamos as placas em incubadora climatizada a $28^{\circ} \mathrm{C}$ e sem iluminação. A cada $12 \mathrm{~h}$ contamos e retiramos as sementes germinadas, com protusão radicular igual ou maior a $2 \mathrm{~mm}$ (Brasil, 1992). Quebramos a dormência das sementes de corda-de-viola com escarificação química ( $\mathrm{H}_{2} \mathrm{SO}_{4} \mathrm{PA}$, durante 4 minutos).

Crescimento inicial - Esterilizamos placas de Petri $(14 \mathrm{~cm})$ forradas com papel filtro e adicionamos 10 plântulas (prégerminadas em água destilada por 48 horas, $28{ }^{\circ} \mathrm{C}$ ) com radículas de $3 \pm 1 \mathrm{~mm}$ (Fase I) e de $5 \pm 1 \mathrm{~mm}$ (Fase II) e $12 \mathrm{~mL}$ do tratamento. Colocamos as placas em incubadora climatizada a $28{ }^{\circ} \mathrm{C}$, com fotoperíodo de 12 horas. Após cinco dias, medimos o comprimento da parte aérea e a raiz de cada plântula.

Potencial osmótico e pH - Determinamos o potencial osmótico dos extratos e convertemos os valores obtidos em $\mu \circ \mathrm{osm}^{-1}$ em MPa utilizando a fórmula: $1 \mu \mathrm{osm} \mathrm{kg}{ }^{-1}=0,00832 \times \mathrm{T} \mathrm{MPa}$; onde $\mathrm{T}=$ temperatura em $\mathrm{K}$. Baseado nos valores obtidos em MPa, montamos um controle com os potenciais $-0,1$, $-0,2,-0,3$ e -0,4 MPa utilizando Polietilenoglicol 6000 (PEG-6000) com a mesma metodologia descrita anteriormente. Determinamos também o pH dos extratos.

Análise dos dados - Utilizamos um delineamento experimental inteiramente casualizado com cinco réplicas em ambas as fases. Para todos os tratamentos fizemos testemunhas com água destilada. Calculamos a porcentagem e o tempo médio de germinação para cada réplica. Analisamos os dados da porcentagem e tempo médio de germinação com o teste Kruskal-Wallis e pós teste Dunn 5\%, e os dados de crescimento com ANOVA e pós teste Tukey 5\%.

\section{Resultados}

Todas as concentrações do extrato de folha reduziram significativamente a porcentagem de germinação das sementes de alface, e quanto mais concentrados, maiores os efeitos inibitórios. Os extratos de bainha diminuíram a porcentagem de germinação (\% G) nas concentrações $50 \%, 75 \%$ e $100 \%$, e o extrato de raiz apenas na concentração 100\%. O tempo médio de germinação (Tmg) de alface foi maior nos extratos de folha e raiz a partir de $25 \%$ e de bainha a partir de $50 \%$ (tabela 1 ).

As sementes de gergelim foram mais inibidas pelos extratos de folha, que reduziram a $\% \mathrm{G}$ a partir da concentração $25 \%$. Assim como, pelas concentrações $75 \%$ e $100 \%$ de bainha e raiz. O Tmg do gergelim foi afetado em concentrações maiores que $75 \%$ nos extratos de bainha, $50 \%$ nos extratos de raiz e por todos os extratos de folha (tabela 1).

Com exceção dos extratos de folha $12,5 \%$ e bainha $25 \%$, todos os extratos diminuíram significativamente a $\% \mathrm{G}$ de rabanete. Para o Tmg, houve aumento para extrato de bainha e raiz nas concentrações $75 \%$ e $100 \%$, e para folha acima de 12,5\% (tabela 1).

Desconsideramos os valores de Tmg nas réplicas em que nenhuma semente germinou, e o Tmg do tratamento que apresentou três ou mais réplicas sem germinação (lacunas na tabela 1).

Os extratos de C. americanum inibem também crescimento inicial das espécies olerícolas. As raízes das plântulas de alface foram inibidas por todas as concentrações dos extratos de folha e raiz, e pelo extrato de bainha nas concentrações maiores que $25 \%$. Apenas o extrato de raiz nas concentrações menores que $75 \%$ não inibiram significativamente a parte aérea das plântulas de alface (tabela 1). Atribuímos valor zero para plântulas mortas ou inviáveis (raízes enegrecidas, plântulas atacadas por fungos ou apodrecidas). Todas 
Tabela 1. Porcentagem e tempo médio de germinação das sementes e crescimento inicial das plântulas de alface, rabanete e gergelim submetidas à ação de diferentes extratos de Crinum americanum. Médias seguidas pelas mesmas letras, maiúsculas para concentração e minúsculas para as partes da planta, não diferem entre si pelo teste Kruskal-Wallis com pós teste de Dunn 5\% para porcentagem e tempo médio de germinação; e pelo teste ANOVA com pós teste de Tukey 5\% para o crescimento inicial. As lacunas (-) correspondem aos tratamentos cujo tempo médio de germinação das sementes não foi calculado.

Table 1. Percentage and germination average time of the seeds and initial growth of the seedings of lettuce, radish and sesame submitted to different extract concentrations of Crinum americanum. The averages followed by the same letters, capital for concentration and lower case for the parts of the plant, don't differ by Kruskal-Wallis with Dunn 5\% pos test for percentage and average time and by ANOVA with Tukey 5\% pos test for initial growth. The lacunes (-) correspond to the treatments whose average time of germination wasn't calculated.

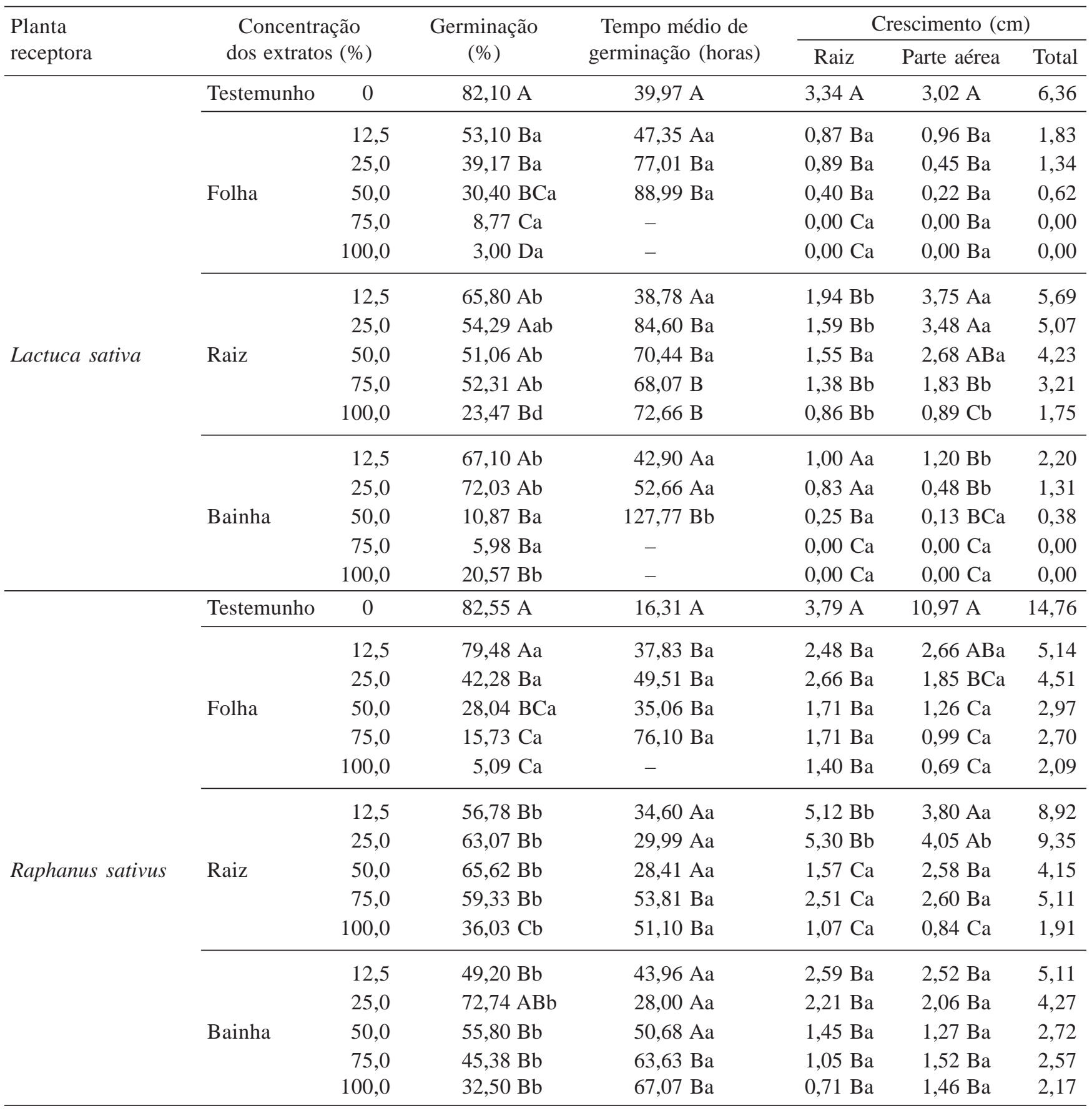


continuação

\begin{tabular}{|c|c|c|c|c|c|c|c|}
\hline \multirow{2}{*}{$\begin{array}{l}\text { Planta } \\
\text { receptora }\end{array}$} & \multirow{2}{*}{\multicolumn{2}{|c|}{$\begin{array}{c}\text { Concentração } \\
\text { dos extratos (\%) }\end{array}$}} & \multirow{3}{*}{$\begin{array}{c}\text { Germinação } \\
(\%)\end{array}$} & \multirow{3}{*}{$\begin{array}{c}\text { Tempo médio de } \\
\text { germinação (horas) }\end{array}$} & \multicolumn{3}{|c|}{ Crescimento $(\mathrm{cm})$} \\
\hline & & & & & \multirow{2}{*}{$\frac{\text { Raiz }}{4,49 \mathrm{~A}}$} & \multirow{2}{*}{$\begin{array}{c}\text { Parte aérea } \\
2,54 \mathrm{~A}\end{array}$} & \multirow{2}{*}{$\begin{aligned} \text { Total } \\
7,03\end{aligned}$} \\
\hline & Testemunho & 0 & & & & & \\
\hline & & 12,5 & $75,00 \mathrm{Aa}$ & $36,40 \mathrm{Aa}$ & $0,75 \mathrm{Ba}$ & $1,50 \mathrm{ABa}$ & 2,25 \\
\hline & & 25,0 & $56,78 \mathrm{Ba}$ & $69,61 \mathrm{Ba}$ & $0,20 \mathrm{Ba}$ & $1,10 \mathrm{Ba}$ & 1,30 \\
\hline & Folha & 50,0 & $40,71 \mathrm{Ba}$ & 73,98 Ba & $0,58 \mathrm{Ba}$ & $1,10 \mathrm{Ba}$ & 1,68 \\
\hline & & 75,0 & $14,83 \mathrm{Cab}$ & $116,40 \mathrm{Ba}$ & $0,27 \mathrm{Ba}$ & $0,85 \mathrm{Ba}$ & 1,12 \\
\hline & & 100,0 & $5,79 \mathrm{Ca}$ & - & $0,04 \mathrm{Ba}$ & $0,75 \mathrm{Ba}$ & 0,79 \\
\hline & & 12,5 & $81,8 \mathrm{Ab}$ & $36,74 \mathrm{Aa}$ & $1,77 \mathrm{Ba}$ & 2,09 Aa & 3,86 \\
\hline & & 25,0 & $77,98 \mathrm{Ab}$ & 47,26 Аa & $1,70 \mathrm{Ba}$ & 1,86 Аa & 3,56 \\
\hline \multirow[t]{8}{*}{ Sesamum indicum } & Raiz & 50,0 & $65,94 \mathrm{Aa}$ & $64,69 \mathrm{Ba}$ & $1,22 \mathrm{Ba}$ & $1,64 \mathrm{Aa}$ & 2,86 \\
\hline & & 75,0 & $28,96 \mathrm{Ba}$ & $95,86 \mathrm{Ba}$ & $0,97 \mathrm{Ba}$ & $1,26 \mathrm{Ba}$ & 2,23 \\
\hline & & 100,0 & $12,51 \mathrm{Ba}$ & - & $0,89 \mathrm{Ba}$ & $1,00 \mathrm{Ba}$ & 1,89 \\
\hline & & 12,5 & $75,00 \mathrm{Aa}$ & $36,00 \mathrm{Aa}$ & $3,70 \mathrm{Ab}$ & $1,37 \mathrm{Aa}$ & 5,07 \\
\hline & & 25,0 & $65,01 \mathrm{Aa}$ & $54,50 \mathrm{Aa}$ & $0,77 \mathrm{Bb}$ & $0,95 \mathrm{Ba}$ & 1,72 \\
\hline & Bainha & 50,0 & $66,94 \mathrm{Ab}$ & 71,94 Aa & $0,13 \mathrm{Ba}$ & $0,25 \mathrm{Bb}$ & 0,38 \\
\hline & & 75,0 & $2,10 \mathrm{Ba}$ & - & $0,00 \mathrm{Cb}$ & $0,03 \mathrm{BCb}$ & 0,03 \\
\hline & & 100,0 & 7,37 $\mathrm{Ba}$ & - & $0,00 \mathrm{Cb}$ & $0,00 \mathrm{Cb}$ & 0,00 \\
\hline
\end{tabular}

as plântulas de alface foram consideradas mortas ou inviáveis no extrato de folha e bainha $100 \%$. A testemunha apresentou viabilidade total.

Todos os extratos inibiram significativamente o crescimento das raízes das plântulas de gergelim, exceto o extrato de bainha $12,5 \%$. A parte aérea dessas plântulas foi inibida por extratos de raiz acima de $50 \%$ e de folha e bainha acima de $12,5 \%$ (tabela 1). Na concentração $75 \%$ e $100 \%$ do extrato de bainha, nenhuma plântula sobreviveu.

O rabanete sofreu inibição das raízes sob todos os extratos e a parte aérea, sob extratos com concentrações maiores que $50 \%$, quando comparados com a testemunha (tabela 1). Mais da metade das plântulas foi considerada morta ou inviável no extrato de raiz $100 \%$.

Mesmo quando consideradas viáveis, as plântulas de espécies olerícolas foram cada vez menos saudáveis com o aumento da concentração dos extratos. Comparados à testemunha, apresentaram raízes menos vigorosas, muitas vezes amarronzadas e com manchas nos cotilédones.

As sementes de picão-preto e corda-de-viola foram as mais sensíveis aos extratos (tabela 2). O extrato $5 \%$ afetou a \% G destas espécies. O Tmg de todas as espécies aumentou com esse extrato. O crescimento inicial de todas as espécies invasoras foi inibido significativamente pelos extratos de C. americanum, reduzindo o comprimento da raiz e da parte aérea das plântulas.

O potencial osmótico dos extratos variou entre - 0,1 e -0,36 MPa e o pH, entre 5,1 e 6,1. O controle osmótico
$(-0,1,-0,2,-0,3$ e -0,4 MPa) não afetou significativamente a germinação ou afetou menos que o extrato correspondente.

A relação massa fresca/seca de $C$. americanum é de 15,2, 11,77 e 7,83 para folha, raiz e bainha, respectivamente. Portanto, o extrato $100 \%$ utilizando material vegetal fresco equivale em massa seca às concentrações de $4,9 \%, 6,3 \%$ e $9,5 \%$.

\section{Discussão}

Diversos estudos mostraram que o potencial osmótico do extrato, e não seus componentes químicos, afeta a germinação (Wardle et al. 1992, (Haugland \& Brandsaeter 1996). Como os controles osmóticos, quando comparados com os extratos de mesma osmolaridade, apresentavam ou ausência ou inibição significativamente menor, descartamos a influência da osmolaridade dos extratos nos resultados observados. Souza Filho et al. (1996) propõem que são necessários valores de pH extremos para influenciar significativamente a germinação. Correia et al. (2005) não detectaram influência na germinação utilizando tratamentos entre pH 4,5 e 8,5. Assim descartamos a influência do pH sobre a germinação e crescimento inicial das plântulas testadas.

Como a \% G é menos sensível que o Tmg, este é um indicativo importante das atividades alelopáticas (Ferreira \& Áquila 2000, Peres et al. 2004). Os extratos 
Tabela 2. Porcentagem e tempo médio de germinação e crescimento de sementes de capim-arroz, picão-preto e corda-deviola submetidas à ação de extratos de folha de Crinum americanum. Médias seguidas pelas mesmas letras, para cada espécie receptora, não diferem entre si pelo teste Kruskal-Wallis com pós teste de Dunn 5\% para porcentagem e tempo médio de germinação; e pelo teste ANOVA com pós teste de Tukey 5\% para o crescimento.

Table 2. Percentage and average time of germination of the seeds and initial growth of the seedings of Echinochloa crussgalli, Ipomoea grandifolia and Bidens pilosa submitted to different extract concentrations of Crinum americanum. The averages followed by the same letters don't differ by Kruskal-Wallis with Dunn 5\% pos test for percentage and average time and by ANOVA with Tukey 5\% pos test for initial growth.

\begin{tabular}{lcccccc}
\hline Planta & \multirow{2}{*}{$\begin{array}{c}\text { Concentração } \\
\text { receptora }\end{array}$} & $\begin{array}{c}\text { Germinação } \\
\text { dos extratos }\end{array}$ & $\begin{array}{c}\text { Tempo médio de } \\
\text { germinação (horas) }\end{array}$ & Raiz & Parte aérea & Total \\
\hline Capim-arroz & $0 \%$ & $63,3 \mathrm{a}$ & $77,3 \mathrm{a}$ & $1,95 \mathrm{a}$ & $1,43 \mathrm{a}$ & $3,38 \mathrm{a}$ \\
& $1 \%$ & $60,0 \mathrm{a}$ & $83,0 \mathrm{a}$ & $1,04 \mathrm{~b}$ & $1,74 \mathrm{~b}$ & $2,78 \mathrm{~b}$ \\
& $5 \%$ & $49,3 \mathrm{a}$ & $135,9 \mathrm{~b}$ & $0,82 \mathrm{c}$ & $1,53 \mathrm{ab}$ & $2,35 \mathrm{c}$ \\
Picão-preto & $0 \%$ & $82,0 \mathrm{a}$ & $68,9 \mathrm{a}$ & $2,26 \mathrm{a}$ & $2,00 \mathrm{a}$ & $4,26 \mathrm{a}$ \\
& $1 \%$ & $88,6 \mathrm{a}$ & $61,9 \mathrm{a}$ & $1,35 \mathrm{~b}$ & $1,59 \mathrm{~b}$ & $2,90 \mathrm{~b}$ \\
& $5 \%$ & $10,6 \mathrm{~b}$ & $135,6 \mathrm{~b}$ & $0,22 \mathrm{~b}$ & $0,75 \mathrm{~b}$ & $0,97 \mathrm{c}$ \\
Corda-de-viola & $0 \%$ & $86,0 \mathrm{a}$ & $38,15 \mathrm{a}$ & $2,68 \mathrm{a}$ & $2,50 \mathrm{a}$ & $5,10 \mathrm{a}$ \\
& $1 \%$ & $80,6 \mathrm{a}$ & $53,68 \mathrm{ab}$ & $0,90 \mathrm{~b}$ & $1,68 \mathrm{~b}$ & $2,50 \mathrm{~b}$ \\
& $5 \%$ & $10,0 \mathrm{~b}$ & $100,3 \mathrm{~b}$ & $0,30 \mathrm{c}$ & $1,17 \mathrm{c}$ & $1,47 \mathrm{c}$ \\
\hline
\end{tabular}

de $C$. americanum reduziram a porcentagem e crescimento inicial e aumentaram o Tmg de todas as espécies olerícolas testadas, indicando um potencial alelopático. Os bioensaios com plantas invasoras de culturas reforçam esses indícios, com a redução da porcentagem e atraso no tempo médio de germinação de picão-preto e corda-de-viola e atraso no Tmg de capim-arroz.

As três espécies invasoras estudadas apresentaram inibição do crescimento inicial sob ação dos extratos. Diversos trabalhos atestam que os efeitos dos aleloquímicos sobre o crescimento inicial refletem em um atrofiamento das partes da plântula (Peres et al. 2004, Aires et al. 2005, Maraschin-Silva \& Áquila 2006). Plântulas de olerícolas inviáveis, como as observadas neste trabalho, também foram detectadas por Oliveira et al. (2004).

Embora seja difícil comparar trabalhos sobre alelopatia devido à grande variedade de metodologias utilizadas, os extratos do $C$. americanum conseguem inibir a germinação em menores concentrações que outras espécies normalmente encontradas na literatura (Soares 2000, Áquila 2000, Pires et al. 2001, Maraschinin-Silva \& Aquila 2005).

Em linhas gerais, a folha é o órgão da planta mais ativo metabolicamente. É razoável que elas apresentem maior diversidade de aleloquímicos. Jacobi \& Ferreira (1991) encontraram efeitos alelopáticos mais efetivos nos extratos de folhas de Mimosa bimucronata (DC). Aires et al. (2005) trabalhando com Solanum lycocarpum também chegaram a essa conclusão.
O alcalóide licorina, poderoso inibidor do crescimento de plantas superiores e algas (Garuccio \& Arrigoni 1989), está presente em abundância nas folhas $C$. americanum, mas é raro em suas raízes (Trimino et al. 1987). Isso explica, pelo menos em parte, o efeito inibitório dos extratos de $C$. americanum $\mathrm{e}$ as diferenças encontradas entre raízes e folhas.

Além dos efeitos diretos da redução da porcentagem de geminação, que ocorreu nas sementes picão-preto e corda-de-viola, os extratos de C. americanum, em linhas gerais, ao atrasarem a germinação das sementes e reduzirem o crescimento inicial das plântulas, podem favorecer as culturas até que estas alcancem um tamanho menos suscetível à competição com as plantas invasoras. Assim sendo, acreditamos que o $C$. americanum tem grande possibilidade de se tornar uma planta doadora para a elaboração de um herbicida verde.

Agradecimentos - Ao Conselho Nacional de Desenvolvimento Científico e Tecnológico (CNPq), pela bolsa de mestrado concedida ao primeiro autor.

\section{Referências bibliográficas}

AIRES, S.S., FERREIRA, A.G. \& BORGHETTI, F. 2005. Efeitos alelopáticos de folhas e frutos de Solanum lycocarpum A. St.-Hil. (Solanaceae) na germinação e crescimento de Sesamum indicum L. (Pedaliaceae) em solo sob três temperaturas. Acta Botanica Brasilica 19:339-344. 
ALI, A.A., El SAYED, H.M., ABDALLAH, O.M. \& STEGLICH, W. 1986. Oxocrinine and other alkaloids from Crinum americanum. Phytochemistry 25:23992401.

ALI, A.A., El SAYED, H.M., ABDALLAH, O.M. \& STEGLICH, W. 1988. Flavans from Crinum americanum. Pharmazie 43:295-296.

ÁQUILA, M.E.A. 2000. Efeito alelopático de Ilex paraguaiensis A. St.-Hil. na germinação e crescimento inicial de Lactuca sativa L. Iheringia, Série Botânica 53:51-66.

BRASIL. 1992. Ministério da Agricultura e Reforma Agrária. Regras para análise de sementes. SNDA/DNDV/CLAV, Brasília.

CORREIA, N.M., CENTURION, M.A.P.C \& ALVES, P.L.C.A. 2005. Influência de extratos aquosos de sorgo sobre a germinação e o desenvolvimento de plântulas de soja. Ciência Rural, Santa Maria, 35:498-503.

DUKE, S.O., DAYAN, F.E, RAMAGNANI, J.G., RIMANDO, A.M. 2000. Natural products as sources of herbicides: current status and future trends. Weed Research 40: 99-111.

ERHARD, D. \& GROSS, E.M. 2006. Allelopathic activity of Elodea canadensis and E. nuttallii against epithytes and phytoplankton. Aquatic Botany 85:203-211.

INDERJIT \& BHOWMIK, P.C. 2002. The importance of allelochemicals in weed invasiveness and the natural suppression. In Chemical Ecology of plant: allelopathy of aquatic and terrestrial ecosystems (Inderjit \& A.U. Mallik, eds.). Birkhauser Verlag AG, Basal. p.188-197.

FERREIRA, A.G. \& ÁQUILA, M.E.A. 2000. Alelopatia: uma área emergente da ecofisiologia. Revista Brasileira de Fisiologia Vegetal 12(Edição especial):175-204.

GARUCCIO, L. \& ARRIGONI, O. 1989. Various sensitivities of yeasts to lycorine. Bollettino della Societa Italiana di Biologia Sperimentale 65:501-508.

GROSS, E.M. 2003. Allelopathy of aquatic autotrophs. Critical Reviews. Plant Science 22:313-339.

HAUGLAND, E. \& BRANDSAETER, L. 1996. Experiments on bioassay sensitivity in the study of allelopathy. Journal of Chemical Ecology 22:1845-1859.

HILT, S. 2006. Allelopathic inhibition of epiphytes by submerged macrophytes. Aquatic Botany 85:252-256.

HUANG, S., WANG, W., MA, K., ZHOU, H., XU, Y., WU, H., SUN, W., YANG, S., HUANG, H.,Z., WU, H. \& YU, S. 1999. Allelochemicals from root exudates and extracts of water hyacinth Eichornia crassipes. In: Recent advances in allelopathy I (F.A.Mácias, J.C.G. Galindo, J.M.G. Molinillo \& H.G. Cutler, eds.). p.197-204.

JACOBI, U.S. \& FERREIRA, A.G. 1991. Efeitos alelopáticos de Mimosa bimucronata (DC) sobre espécies cultivadas. Pesquisa Agropecuária Brasileira 26:935-943.
MAYER, L.A., POWELL, R., JENKINS, C., LOOKWOOD, J., FENN, K. \& PIMM, S. 1998. Identifying Cape Sable Seaside-Sparrow (Ammodramus maritimus mirabilis) habitat from satellite images. http://www.nicholas. duke.edu acess em 06/02/07.

MARASCHIM-SILVA, F. \& ÁQUILA, M.E.A. 2006. Potencial alelopático de espécies nativas na germinação e crescimento inicial de Lactuca sativa L. (Asteraceae). Acta Botanica Brasilica 20:61-69.

OLIVEIRA, E.A. \& RIBEIRO-COSTA, C.S. 2006. Coleópteros de uma ilha estuarina da Lagoa dos Patos, Rio Grande do Sul, Brasil. Dissertação de mestrado, Universidade Federal do Paraná, Curitiba.

OLIVEIRA, S.C.C., FERREIRA, A.G. \& BORGHETTI, F. 2004. Efeito alelopático de folhas de Solanum lycocarpum A. St.Hil. (Solanaceae) na germinação e crescimento de Sesamum indicum L. (Pedaliaceae) sob diferentes temperaturas. Acta Botanica Brasilica 18:401-406.

PERES, M.T.L.P., SILVA, L.B, FACCENDA, O. \& HESS, S. C. 2004. Potencial alelopático de espécies de Pteridaceae (Pteridophyta). Acta Botanica Brasilica 18:723-730.

PIRES, N.M., PRATES, H.T., FILHO, I.A.P., OLIVEIRA JUNIOR, R.S. \& FARIA, T.C.L. 2001. Atividades alelopáticas da leucena sobre espécies de plantas daninhas. Scientia Agricola 58:61-65.

PHILLIPS, G.L., EMINSON, D.F. \& MOSS, B. 1978. A mechanism to account for macrophyte decline in progressively eutrophicated freshwater. Aquatic Botany 4:103-126.

PUTNAM, R.A., 1983. Allelopathy: a break in weed control? American Fruit Grower 103:10.

SANTOS, S. \& RESENDE, M.O.O. 2008. Avaliação do potencial herbicida de compostos secundários na germinação de sementes de plantas daninhas encontradas em pastagens. Revista Analytica 32:72-78.

SOARES, G.L.G. 2000. Inibição da germinação e do crescimento radicular de alface (CV. "Grand rapids") por extratos aquosos de cinco espécies de Gleicheniaceae. Floresta e Ambiente 7:190-197.

SOUZA FILHO, A.P.S., RODRIGUES, L.R.A. \& RODRIGUES, T.J.D. 1996. Efeitos de extratos aquosos de assa-peixe sobre a germinação de três espécies de braquiária. Planta Daninha 14:93-101.

TRIMINO, Z., IGLESIAS, C., SANCHEZ, L. \& M., SPENGLER, I. 1987. Phytochemical investigation of Crinum americanum. Part I. Major alkaloids in leaves. Revista Cubana de Química 3:57-61.

WARDLE, D.A., NICHOLSON, K.S. \& AHMED, M. 1992. Comparison of osmotic and allelopathic effects of grass leaf extracts on grass seed-germination and radicle elongation. Plant and Soil 140:315-319. 